\title{
Synchronization in reaction-diffusion systems with multiple pacemakers
}

F.E. Nolet, ${ }^{1, \text { a) }}$ J. Rombouts, ${ }^{1, \text { a) }}$ and L. Gelens ${ }^{1, b)}$

Laboratory of Dynamics in Biological Systems, Department of Cellular and Molecular Medicine, University of Leuven, 3000 Leuven, Belgium

(Dated: 8 May 2020)

Spatially extended oscillatory systems can be entrained by pacemakers, regions which oscillate with a higher frequency than the rest of the medium. Entrainment happens through waves originating at a pacemaker. Typically, biological and chemical media can contain multiple pacemaker regions which compete with each other. In this paper we perform a detailed numerical analysis of how wave propagation and synchronization of the medium depend on the properties of these pacemakers. We discuss the influence of the size and intrinsic frequency of pacemakers on the synchronization properties. We also study a system in which the pacemakers are embedded in a medium without any local dynamics. In this case, synchronization occurs if the coupling determined by distance and diffusion is strong enough. The transition to synchronization is reminiscent of systems of discrete coupled oscillators.

PACS numbers: 05.45.-a, 05.45.Xt, 89.75.Fb

Keywords: Reaction-diffusion systems, synchronization, pacemakers, oscillatory medium, coupled oscillators

\begin{abstract}
How do oscillatory chemical and biological systems synchronize their dynamics over large distances? Pacemaker regions, which oscillate faster than the surrounding medium, send out waves which can coordinate the entire medium. Here, we address what happens when multiple pacemakers compete with each other. We perform a detailed numerical investigation of a reactiondiffusion system based on coupled FitzHughNagumo oscillators. We determine when and how pacemakers synchronize depending on their size, oscillation frequency, and type of coupling.
\end{abstract}

\section{INTRODUCTION}

Oscillation and synchronization are widely observed phenomena, from the synchronous flashing of fireflies to the target patterns observed in a dish filled with chemical reagents for the Belousov-Zhabotinsky (BZ) reaction. ${ }^{1}$ In spatially extended oscillatory systems, synchronization is often obtained through the action of pacemakers, which entrain the surrounding medium. In chemical systems such as the BZ reaction or the oxidation of $\mathrm{CO}$ on $\mathrm{Pt}(110),{ }^{2}$ these pacemakers typically appear through impurities such as dust particles or imperfections in the dish. However, they can also be induced, for example to suppress chaotic behavior. ${ }^{3}$ Biological examples where pacemakers play a role include populations of Dictyostelium discoideum, ${ }^{4}$ oscillations in Xenopus laevis cell extracts $^{5}$ or cardiac tissue, where they play a role in arrhythmias. ${ }^{6}$

\footnotetext{
a) Contributed equally

b) Electronic mail:

http://www.gelenslab.org
}

A large number of mathematical models have been proposed and analyzed in order to explain how these systems generate their typical synchronizing behavior. ${ }^{7}$ For systems which are continuous in space, as is often the case for oscillating chemical or biological media, mathematical modeling usually takes the form of reaction-diffusion equations. It has been long established that inhomogeneities in an oscillating system can generate outgoing waves which entrain the entire medium. ${ }^{8,9}$ Those inhomogeneities act to locally increase the oscillation frequency and are usually called pacemakers. The early analytical work also showed that when two pacemakers are present in a medium, it is the fastest one that finally wins and overtakes the entire medium. Studies that explicitly address multiple pacemakers are more rare. Mikhailov and Engel studied multiple pacemakers in the complex Ginzburg-Landau equation. ${ }^{10}$ In their model, pacemakers are regions with random radius scattered in an oscillatory medium. All pacemakers share a frequency, slightly larger than that of the surrounding medium. The authors show analytically that in the end, the entire medium will be synchronized and the process will happen through competing expanding target patterns. Tönjes and Blasius went a step further and studied analytically the case in which frequencies are randomly distributed over the medium. ${ }^{11}$ They apply a perturbation method to the nonlinear phase equation ${ }^{9}$ and obtain the synchronized state on different topologies. Both the nonlinear phase equation and the complex Ginzburg-Landau equation are often used as the starting point for a study of inhomogeneities in oscillatory media. This is mostly due to their analytical tractability. However, both equations are approximate descriptions obtained by a perturbation argument. Despite their usefulness, they do not constitute a realistic model for most real chemical and biological oscillatory systems. Those are often better represented by relaxation oscillators built on systems with multiple steady states. ${ }^{12}$ Prototypical examples are Van der Pol or FitzHugh-Nagumo type models. Relaxation oscilla- 
tions also appear in the $\mathrm{BZ}$ reaction and its models. One notable numerical experiment with multiple competing pacemakers in the BZ reaction was performed by Bugrim et al. ${ }^{13}$ They studied randomly scattered pacemakers in a medium that oscillates with BZ kinetics. Interestingly, they defined the power of a pacemaker as the product of its size and the difference of a reaction rate between the pacemaker and the surrounding medium. This rate essentially determines the local frequency. The numerical model was able to explain the appearance of new centers of target patterns in an experimental setup.

Most studies of synchronization through a pacemaker consider the whole medium to be oscillatory, with a pacemaker being a small region of higher frequency. However, in some systems the pacemakers are the only oscillatory parts, and the space between them has other dynamics. For example, recent research in embryos of the fruit fly Drosophila melanogaster has shown that only the nuclei in the syncytium oscillate and propagate their influence in a non-oscillatory medium. ${ }^{14}$ More recent experimental work on the BZ reaction has gone beyond the classical system in a dish. Examples include square oscillatory regions in an otherwise excitable medium, made by imposing light patterns on a gel, ${ }^{15}$ or using discrete beads ${ }^{16-18}$ or aqueous droplets ${ }^{19}$ immersed in oil. On those beads or in the droplets, the system is oscillatory whereas in the medium in between, chemicals can merely diffuse. Depending on which chemicals can easily diffuse, different dynamics are observed. Taylor et al. ${ }^{16}$ study beads with BZ reagents in a well-mixed medium and see a transition to synchrony when the density of oscillators crosses a threshold, which they relate to the concept of quorum sensing in biological systems. Systems in which the coupling is mediated by the geometry, and more particularly the distance between pacemakers, include the one by Fukuda et al., ${ }^{18}$ who considered a system in which one bead oscillates faster and acts as a pacemaker. They control the coupling strength by modifying the distance between the beads and find entrainment for small distances. Li et al. ${ }^{19}$ similarly examined how the coupling strength between aqueous droplets depends on their distance, droplet size and concentration of malonic acid, which influences the period of the oscillation. Recently, a synthetic biological system has been created in which different genetic oscillators interact through purely diffusive channels. ${ }^{20}$ Tayar et al. managed to adjust coupling strength by varying the geometry of their system of artificial cells and show a transition to synchrony for large coupling. Another example where coupling occurs through a medium with different dynamics from the oscillating systems is the dynamics of candles put close to each other, whose flames can oscillate in synchrony or antiphase depending on the distance between them. ${ }^{21}$

This paper aims to fill some gaps in the study of coupled oscillators in spatially extended systems. Since the fastest oscillator entrains the medium, we want to quantify what makes a pacemaker fastest, depending on its size and intrinsic frequency. We compare the speed of the waves sent out from such a single pacemaker with those in systems with two pacemakers. Additionally we ask the question what happens when the regions between pacemakers lack local dynamics, but allow free diffusion. What are the synchronization properties of such systems, can we speak of waves and if so, how does their speed compare to the wave speed in an oscillatory medium?

Whereas in most experimental systems, pacemakers are regions of higher frequency that entrain the rest of the medium, this is not always the case, but depends on the dispersion of the medium. In media with negative dispersion, regions of lower frequency can send out waves, and high-frequency regions can act as the center of inward traveling waves. ${ }^{22}$ Inward traveling target patterns, also called antiwaves, have been observed experimentally as well. ${ }^{23}$ It seems that negative dispersion mainly occurs for systems close to a Hopf bifurcation. ${ }^{23}$ Since we consider oscillations of relaxation type in this paper, we will consider pacemakers as regions with a higher frequency.

The structure of the paper is as follows: we introduce the model we use in Section II. Next we simulate the model in different scenarios. First, we examine how a single pacemaker generates waves in an oscillatory medium, and how those waves depend on the properties of the pacemaker. In addition, we embed the pacemaker in a medium without local dynamics, but where the variables can still diffuse freely. This setup influences the pacemaker region and slows down the oscillation (Section III). Secondly, Section IV deals with two pacemakers competing with each other. Depending on the relative size and frequency, one of both will win and entrain the entire medium. When those pacemakers are embedded in a medium without local dynamics, they may still synchronize if they are close enough. Finally, we investigate a system with many pacemakers, with a faster one in the center (Section V). The surrounding oscillators can act as 'repeaters' of the signal emanating from the central pacemaker. We investigate in detail how this system acts with different types of dynamics in the gaps between the pacemaker regions.

\section{THE MODEL}

We perform simulations using the FitzHugh-Nagumo (FHN) equations, which are a prototype for relaxation oscillations. Oscillations of this type are often encountered in chemical and biological oscillating systems. They are characterized by different timescales and fast transitions. We use the following equations:

$$
\begin{aligned}
u_{t} & =D u_{x x}+\tau_{\mu}(x) F(u, v) \\
v_{t} & =D v_{x x}+\tau_{\mu}(x) G(u, v) \\
F(u, v) & =u-u^{3}-v \\
G(u, v) & =\epsilon(u-b v+a) .
\end{aligned}
$$


The model includes a diffusion constant $D>0$ and parameters $a, b \in \mathbb{R}$ and $\epsilon>0$. For small $\epsilon$, the $u$ variable evolves on a faster timescale than $v$ except close to the nullcline $F(u, v)=0$. This nullcline is fixed and determined by a cubic function. The second nullcline $(G(u, v)=0)$ is a straight line determined by the parameters $a$ and $b$. Depending on the parameter values, this system can have bistable, excitable or oscillatory behavior. If not otherwise specified, parameters are set on $(a, b, \epsilon)=(0.5,0,0.01)$. For this set, the system admits limit cycle oscillations. Fig. 1a shows the two nullclines for this parameter set together with the resulting limit cycle. A time series of this limit cycle is shown in Fig. 1 b.

The diffusion constants are the same for both variables. This is different from the original system in which the FHN equations were used: the propagation of an electrical impulse along an axon. ${ }^{24,25}$ In those systems, the slow variable $v$ does not diffuse. Since we use the FHN system as a prototype (bio)chemical oscillator where $u$ and $v$ can both represent the concentrations of a chemical, we work with equal diffusion coefficients.

The function $\tau_{\mu}(x)$, also referred to as frequency function, is used to rescale time locally. Regions in space with a higher value of $\tau_{\mu}$ have a higher oscillation frequency (Fig. 1c). In these pacemaker regions, $\tau_{\mu}$ is increased relative to a fixed background value, chosen to be $\bar{\tau}=5$. In pacemaker regions, $\tau_{\mu}=\bar{\tau}+\Delta \tau$. In our simulations with two or more pacemakers, one pacemaker can have a different intrinsic frequency than the other(s). This pacemaker has $\tau_{\mu}=\bar{\tau}+p \Delta \tau$, i.e. a higher frequency than the other pacemakers if $p>1$ and lower frequency when $p<1$. The intrinsic period of the oscillation with $\tau=5$ is denoted by $T_{0} \approx 55$ (Fig. 1b). In our plots, we rescale time with $T_{0}$.

Pacemakers have a width $w$ and are placed at a distance $d$ from each other. In order to modify the behavior of the medium outside of the pacemaker regions, we introduce the parameter $\mu \in[0,1]$. This parameter alters the frequency function $\tau_{\mu}$ outside pacemaker regions via $\tau_{\mu}=\mu \bar{\tau}$. To lighten the notation, we use $\tau$ instead of $\tau_{\mu}$ from now on, but we will explicitly mention the values of $\mu$ used. For $\mu=1$, the background dynamics are oscillatory, while for $\mu=0$, the system lacks reaction dynamics and there is only diffusion between the pacemakers. Although any parameter which affects the period of the oscillation can be used to introduce an inhomogeneity, we opt for a rescaling of time through the function $\tau$, since this does not change the shape of the limit cycle.

We simulate these equations in Fortran, using a forward Euler method in time and a central difference method in space, on a domain with periodic boundary conditions. All our simulations were done for a total time of $T=20000 \approx 365 T_{0}$. This is large enough to ensure convergence. The time needed for convergence depends on the details and size of the system. Larger domains take more time to synchronize, and systems with $\mu=0$ take longer than systems with $\mu=1$. Typically, the quickest to converge took 10-20 periods and the longest simulations (such as the ones for 21 pacemakers) could take 100-200 periods. We leave the investigation of the influence of domain size, coupling type and pacemaker properties on the transient times for future study, and only consider long-time behavior in this paper. To ensure the validity of our results, we repeated a selected set of simulations in Python, using a pseudo-spectral method. This showed only a minor quantitative deviation for a few simulations, and all our results remained unchanged. For simplicity, we only consider one-dimensional systems. Examples of simulations are shown in a space-time plot in Fig. 1d,e. These are simulations with 21 pacemakers, in an oscillatory medium $(\mu=1$, panel $\mathrm{d})$ and a medium without local dynamics ( $\mu=0$, panel $\mathrm{e})$.

After simulating the model, we detect profiles in $(x, t)$ space. For every position $x$, we calculate the times $t$ where $u$ crosses 0 from below. The resulting curves are essentially curves of constant phase. An example is plotted in Fig. 1d (in orange). Other examples can be seen in Fig. 3d and Fig. 4a. From the slope of these profiles, we can derive the speed of outgoing waves if there are any. Additionally, it allows detection of the wave origin by locating the absolute minimum of such a profile $\left(x_{p}\right.$ in the figure). When the medium has no local dynamics $(\mu=0)$, there might not be points on the curve for every $x$, since $u$ does not always cross zero outside the pacemaker regions. If there is still a wave, the speed can be estimated by considering only the $(x, t)$ points where $x$ is a position in the middle of the pacemaker (orange curve in Fig. 1e).

In the following section, we will first explain how one pacemaker synchronizes a medium and how this synchronization depends on its size and frequency. The section thereafter deals with the case of two pacemakers, followed by a study of a system with 21 pacemakers.

\section{ONE PACEMAKER}

We study one pacemaker of width $w$ in a region of length $L$. Fig. 2a,b show examples of solutions with an oscillatory $(\mu=1)$ and a medium without local dynamics $(\mu=0)$ for a pacemaker of width $w=100$ in a domain of size $L=700$.

When the surrounding medium is oscillatory, waves propagate from the pacemaker outwards (Fig. 2a). The speed of the wave depends on three parameters: its width, its local frequency increase and the diffusion coefficient. Fig. 2c,d show the effects of $\tau, w$ and $D$ on the wave speed. In short, the speed increases with the diffusion, a large pacemaker sends out slower waves, and if the frequency difference with the surrounding medium increases, the waves are also slower. This might seem counterintuitive, since one might associate a strong pacemaker with fast waves. 

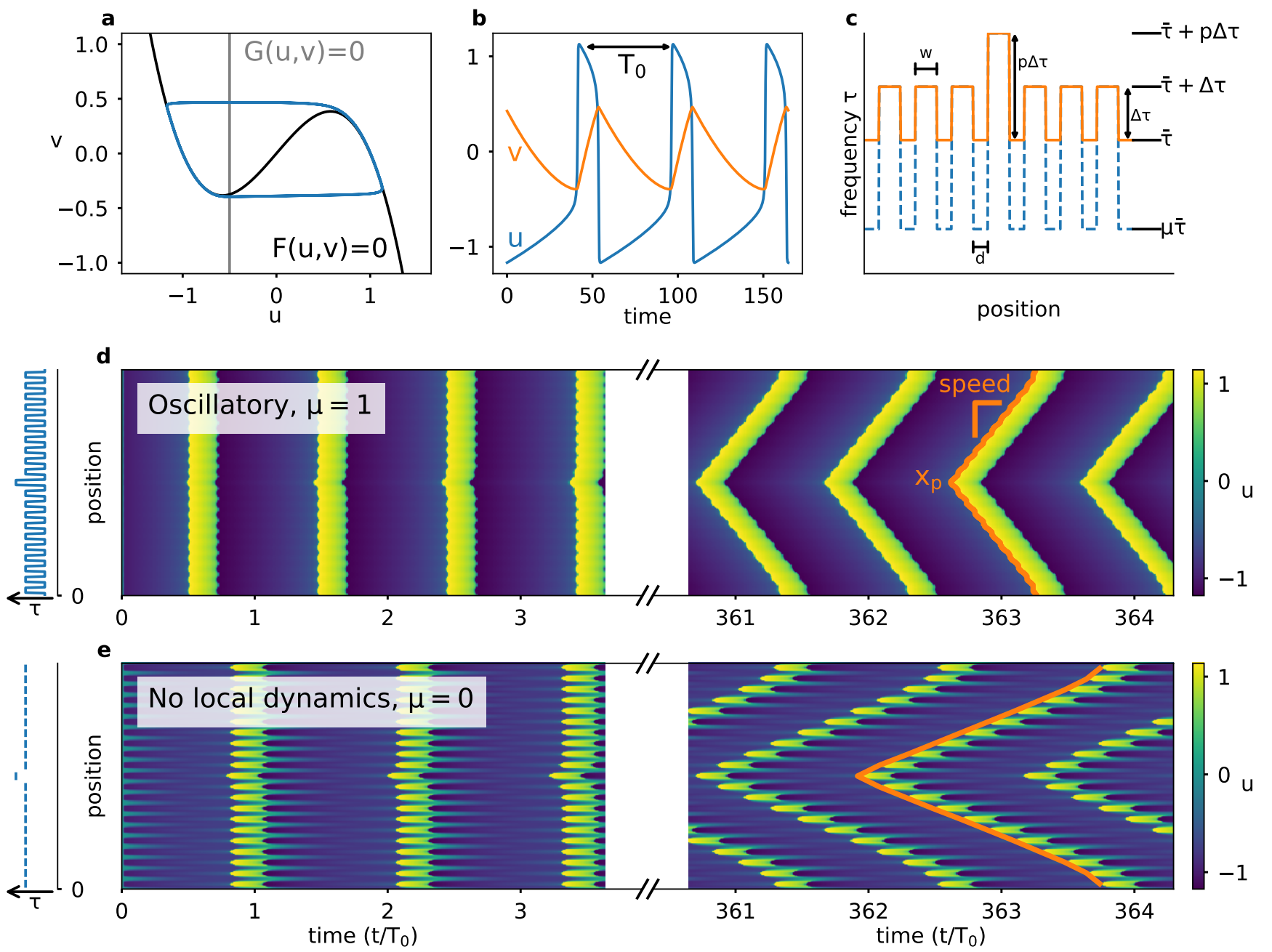

FIG. 1. Setup of the problem. (a) Nullclines $(F(u, v)=0$ black, $G(u, v)=0$ gray) of the system and the limit cycle (blue) of the oscillation in phase space. Parameters $(a, b, \epsilon)=(0.5,0,0.01)$. (b) Time series of $u$ (blue) and $v$ (orange) of the limit cycle. (c) Sketch of frequency function $\tau_{\mu}(x)$ used to define pacemaker regions. Pacemakers have width $w$ and are placed at a distance $d$ from each other. The middle pacemaker has a frequency difference with the baseline $\bar{\tau}$ which is $p$ times the difference of the other pacemakers with $\bar{\tau}$. In this sketch, $p>1$. For clarity this sketch is made for 7 pacemakers $(6+1)$, in our simulations we use $21(20+1)$ pacemakers. (d) Space-time plot of $u$. Approximately the first and last three periods are shown in a simulation of total time $T=20000 \approx 365 T_{0}$. Solution for 21 pacemakers in an oscillating medium $(\mu=1)$ and with parameter values $(p, w, d, D)=(1.5,100,100,80)$. The curve of constant phase in orange is used to calculate the wave speed. The wave starts at the point $x_{p}$, the location of the strongest pacemaker. On the left, tilted: a sketch of the frequency function $\tau(x)$. Initial condition $u=v=0$ everywhere. (e) Similar as (d) but in a surrounding medium without local dynamics: in the gaps between the pacemakers there is only diffusion $(\mu=0)$. In $(\mathrm{d})$ and $(\mathrm{e})$, time is rescaled with the period $T_{0}$ of the oscillation.

In a medium without local dynamics $(\mu=0)$, there are no waves present (Fig. 2b). Close to the pacemaker location damped oscillations appear due to diffusion. Importantly, the period of the pacemaker region itself is significantly affected by its immersion in the medium (Fig. 2e,f). A larger diffusion constant lengthens the pacemaker's period. If the length $L$ of the domain increases, the period initially increases as well, but then stabilizes for larger values of $L$. Intuitively, this could be explained by considering that higher diffusion and a large volume of surrounding medium lead to leaking of $u$ and $v$ into the region outside the pacemaker, slowing down the progression along the limit cycle in the pacemaker region.

\section{TWO PACEMAKERS}

When two pacemakers are present in an oscillatory medium, both of them send out waves as we described in the previous section. If the pacemakers are at a large distance from each other, these target patterns emerge independently and then interact when they meet. After a 


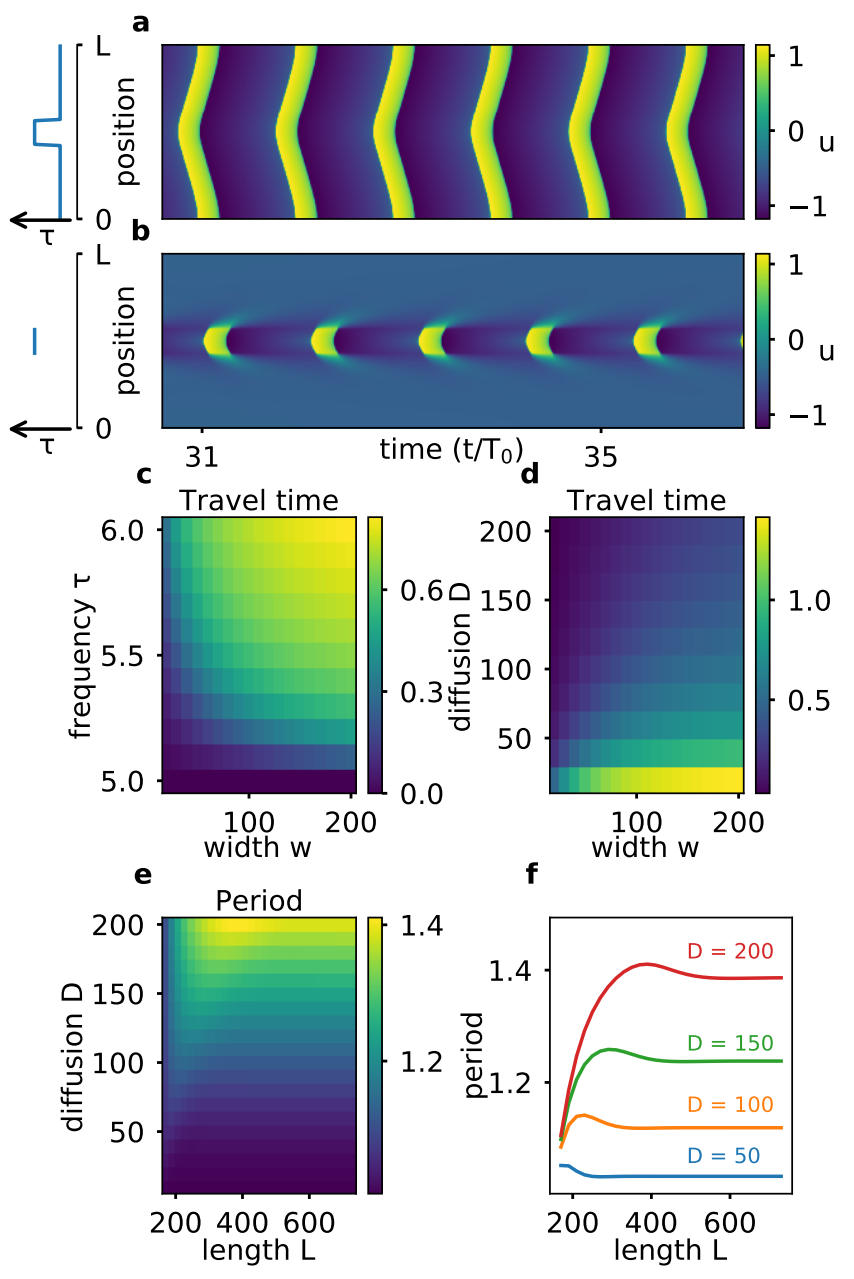

FIG. 2. Analysis for one pacemaker. (a) Space-time plot of $u$ with a single pacemaker with size $w=100$ in a domain of size $L=700$ and diffusion $D=50$. Surrounding medium is oscillatory $(\mu=1)$. (b) Similar as (a) but in a medium without local dynamics $(\mu=0)$. (c) Travel time to the boundary of waves as function of $\tau$ (at pacemaker location) and $w$, in oscillatory surrounding medium $(\mu=1)$. Region size is $L=2000+w$, diffusion $D=100$ and $\bar{\tau}=5$. (d) Travel time to the boundary of waves as function of $D$ and $w$, for $\mu=1$ and $\tau=5.3$ in the pacemaker region compared to $\tau=\bar{\tau}=5$ in the surrounding medium. (e) Period as function of $D$ and $L$ for fixed $w=150$ in a medium without local dynamics $(\mu=0)$. (f) Period as function of $L$ for fixed $w=150$. Plotted for different diffusion constants $D$, for $\mu=0$. In panels (c-f), travel time and period are relative to $T_{0}$.

long time, the whole medium is entrained by the fastest of the two pacemakers. ${ }^{8,9}$ Here we quantify how being the fastest depends on pacemaker width and frequency difference. We consider a system of length $L=4000$ with two pacemakers at a distance $d(\ll L)$ from each other. One pacemaker, $P_{1}$, has $\tau=\bar{\tau}+\Delta \tau$, the second $\left(P_{2}\right) \tau=\bar{\tau}+p \Delta \tau$, i.e. it is $p$ times 'stronger'. In the case of an oscillating medium $(\mu=1)$, both pacemakers initially generate waves, but after a long time one pacemaker takes over the whole medium (Fig. 3a). Similarly to the situation with a single pacemaker, there are no waves present when the surrounding medium has no local dynamics $(\mu=0)$, shown in Fig. 3b.

Which pacemaker wins in an oscillatory medium? The pacemaker that wins is the one which, after a long time, entrains the whole medium and becomes the sole source of target patterns. If the pacemakers have the same size $w$, the pacemaker with the highest intrinsic frequency wins. In our system this is the pacemaker with the highest $\tau$. When the pacemakers have different sizes, there is a trade-off: pacemakers which are intrinsically slower can still win if they compensate by being larger (Fig. 3c). This plot shows the results of varying the frequency and width of $P_{2}$, compared to $P_{1}$ with fixed width and $\tau$. The width of $P_{1}$ is equal to 100 . The colored region shows the values of $w$ and $p$ for which $P_{2}$ wins. Clearly, a larger and faster $P_{2}(w>100, p>1)$ will always overtake the whole medium. However, even slower $(p<1)$ pacemakers can win by being wide enough, and small $(w<100)$ pacemakers can win by compensating in frequency. Interestingly, the properties that make a pacemaker a winner, i.e. which will lead it to overtake the whole medium, are also those properties that slow down the waves it sends out (Fig 2c). The importance of $p$ and $w$ has also been noted by Bugrim et al. ${ }^{13}$ in a model of a Belousov-Zhabotinsky medium, where they use the product of the size and a quantity related to frequency to define the power of a pacemaker. The influence of size and frequency difference of a pacemaker on resulting target patterns has been studied in detail for the complex Ginzburg-Landau equation in 2D. ${ }^{26}$

A second question comes to mind when looking at twopacemaker systems. Once the strongest pacemaker has synchronized the whole medium through outgoing waves, does the weaker pacemaker still somehow influence the wave? In particular, does the presence of the weaker pacemaker speed up or slow down the wave sent out by the dominant one? To investigate this, we use a curve of constant phase of the oscillation in the $(x, t)$-plane, as defined before (Fig. 3d). The local minima of this curve correspond to the positions of pacemakers, with the absolute minimum being the dominant one. We use this curve to calculate the time a signal needs to travel a distance $L^{*}=1000$ both on the left and right of the leading pacemaker. We then compare this with the same value computed for the situation with a single pacemaker with the same width and frequency as the strong one. The results are shown in Fig. 3e. We see that the travel time is decreased when a second pacemaker is added, i.e. the speed of the wave has increased. In other words, having a second pacemaker helps to 'relay' the signal and make its propagation faster. This could be beneficial in biological systems, where synchronization through waves is used, for example, in synchronizing the internal state of a cell. Note that the travel time on the other side of the pacemaker (without 'repeater') is the same as for a single pacemaker. This result holds if the second pacemaker is 

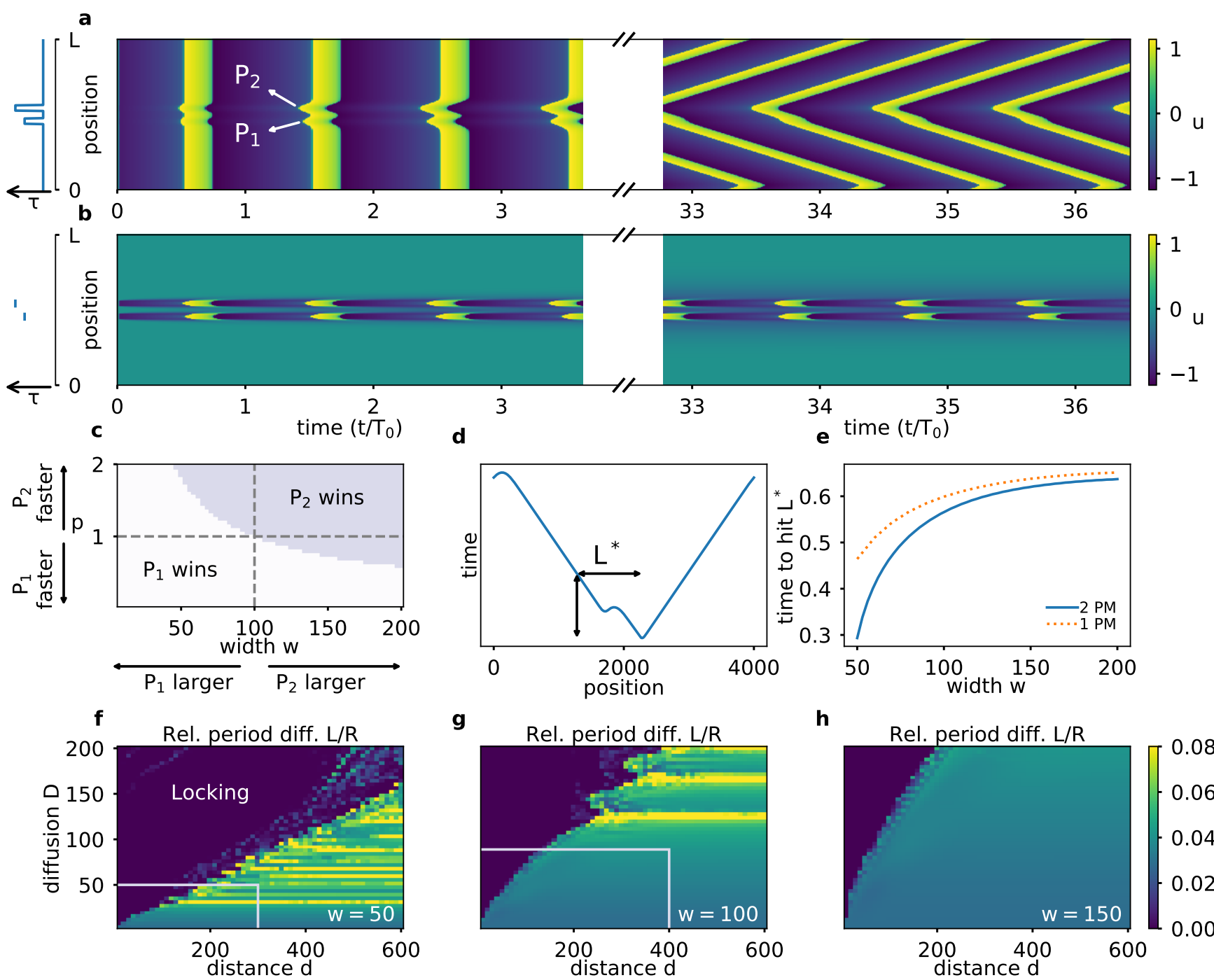

g

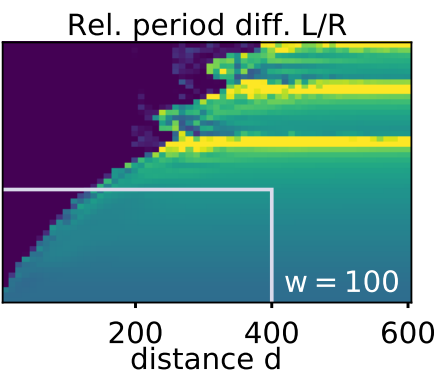

h

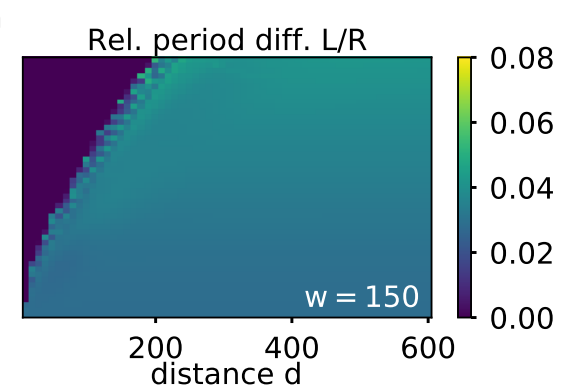

FIG. 3. Analysis for two pacemakers. (a) Space-time plot of $u$ in the model with two pacemakers of width $w=150$ at a distance $d=200$ of each other. Medium is oscillatory $(\mu=1)$ with diffusion $D=50, \bar{\tau}=5$ in the medium and $\tau=5.3$ at the left pacemaker, i.e. $\Delta \tau=0.3$. At the right pacemaker, $\tau=\bar{\tau}+p \Delta \tau$ with in this case $p=1.5$. On the left, tilted: a sketch of the frequency function $\tau(x)$. (b) Similar as (a) but for a medium without local dynamics $(\mu=0)$. (c) The pacemaker that will entrain the medium as function of $p$ and width $w$ of $P_{2}$. The other pacemaker $P_{1}$ has fixed $\tau=\bar{\tau}+\Delta \tau=5.3$ and $w=100$ (indicated by gray dashed lines). Oscillatory medium $(\mu=1)$ with diffusion $D=100$. The pacemakers are at a distance of $d=500$. (d) Example of a curve of fixed phase $(u=0)$. The arrows indicate a distance $L^{*}$ from the dominating pacemaker, and the time it takes for the wave to reach that point. (e) Time (relative to $T_{0}$ ) to travel a distance $L^{*}=1000$ as function of $w$, compared between having two pacemakers (blue) or a single pacemaker (orange dashed). Medium is oscillatory $(\mu=1)$. The value of $\tau=5.6$ for the dominating pacemaker (corresponding to $p=2$ ) and $D=100, d=500$. (f) Relative difference in period of $P_{1}$ and $P_{2}$ of widths $w=50$ for a medium without local dynamics $(\mu=0)$, as function of $D$ and $d$. (g) Similar as (f) but for pacemakers of width $w=100$. (h) Similar as (f,g) but for pacemakers of width $w=150$. The rectangular boxes in panel (f) and $(\mathrm{g})$ show the equivalent regions covered in panels $(\mathrm{g})$ and $(\mathrm{h})$, respectively.

at a large enough distance from the first one. If pacemakers are closer together, they behave more and more like a large, single pacemaker, which would slow down the wave (as discussed in Section III).

Next we look at the situation without local dynamics $(\mu=0)$ and investigate whether the two pacemakers can still entrain or influence each other, even though there are no traveling waves in the medium. For two pacemakers at a large distance $d$ from each other, one expects that they will essentially behave independently and oscillate with different frequencies. This frequency will be lower compared to the $\mu=1$ situation as we have shown for one pacemaker (Fig. 2e,f). When the distance between the two pacemakers is small enough, they can sense and influence each other through the diffusion of $u$ and $v$, and for $d$ below a critical distance, they synchronize. This phe- 
nomenon is shown in Fig. 3f-h, where the relative period difference between the two pacemakers is plotted as function of the diffusion constant $D$ and the distance $d$. This is done for a fixed value $p=1.5$ and different pacemaker widths $w \in\{50,100,150\}$. The dark blue areas correspond to parameter values where the two pacemakers are locked or synchronized, with a relative period difference of (near) zero. It is clear that small distances and large diffusion constants promote synchronization. The small $d$, large $D$ regime intuitively corresponds to stronger coupling, which can induce frequency locking. The locking area decreases in size with increasing widths of the pacemakers, i.e. it is harder to lock for larger pacemakers. Besides this, the plot shows additional structure which is worth mentioning. For specific ranges of $D$, the relative period difference suddenly increases. These are the yellow bands, best visible for pacemaker widths $w=100$ (Fig. 3g). However, it seems that the same happens for $w=50$, only for more and lower values of $D$ making it harder to distinguish the different bands. For the values of $D$ shown here, no such bands occur for $w=150$. They do however show up when the simulations are performed for larger values of $D$. This can be explained by considering the plots with rescaled axes: when plotted as function of $d / w$ and $D / w^{2}$, we find that the middle plot is a zoomed-in version of the left plot, and the right plot a zoomed-in version of the middle plot (indicated by rectangles). The dynamics, therefore, are governed by the relative strength of diffusion and distance, with respect to the pacemakers' width. This is to be expected, given that the rescaling of the axes in these plots corresponds to a rescaling of the space variable in the equations.

\section{MANY PACEMAKERS}

After one and two comes many, so in this final section we consider systems with a large number of oscillators. We expect similar behavior to the two-pacemaker system. This means that for oscillatory $(\mu=1)$ media, we expect the fastest one to entrain the whole medium. For $\mu=$ 0 , synchronization is likely to happen for low distance and large diffusion, whereas we expect to see independent oscillations for larger distances.

We investigate a system of 21 pacemakers with the middle pacemaker $p$ times stronger than the 20 surrounding pacemakers (see Fig. 1c). All pacemakers have the same width $w$ and are spaced a distance $d$ apart. The total domain size is then indirectly defined by $L=$ $21(w+d)$.

In an oscillatory medium $(\mu=1)$, waves propagate outwards from the middle (stronger) pacemaker (Fig. 1d). This is similar to what would happen for a single pacemaker in an otherwise oscillatory medium (Section III). So, what is the added effect of the 20 weaker pacemakers? Fig. 4a shows a phase profile for a system with one, two and 21 pacemakers suitably translated such that the origin of the wave coincides. Each of the simu- lations was done with equal parameters. The addition of a single pacemaker produces a 'dip' in the profile, which means that the signal from the central pacemaker will reach a far-off point faster (see also Fig. 3d,e). Let $\Delta T$ be the gain in travel time from one additional pacemaker, then we hypothesize that the addition of $n$ pacemakers will give a boost of $n \Delta T$. The curve corresponding to this hypothesis is shown as a dashed line in Fig. 4a, and indeed this gives a good approximation of the situation with 21 pacemakers (or $n=10$ additional pacemakers on one side). We conclude that additional (weaker) pacemakers act as 'repeaters' and speed up the wave. Importantly, if one were to measure the slope of the line between the dips, it would be the same for all three situations.

When the region between pacemakers is not oscillatory, the distance between them determines the behavior of the system. In Fig. 1e, it was clear that the middle pacemaker can also send out waves if the distance between pacemakers is small enough. We quantify this by letting the parameter $\mu$ go from 1 (oscillatory) to 0 (no local dynamics) and detect whether the different pacemaker regions are synchronized, and if they are, what the apparent wave speed is (Fig. 4b). For small distances $d$, waves are observed for all values of $\mu$. In this regime, higher values of $\mu$ correspond to faster waves. For larger distances $d$, there is a jump towards desynchronization if $\mu$ is below a threshold. For these large distances, the system goes from wave propagation at $\mu=1$ to independent oscillators at $\mu=0$. In between, a large range of different types of behavior is possible. Fig. 4d shows some snapshots with curves of constant phase.

For $\mu=0$, both distance and diffusion determine the coupling strength and the locking behavior of the pacemaker regions. Locking and waves appear if the distance is small enough which corresponds to stronger coupling. Strong coupling can equally be achieved by increasing the diffusion constant $D$. Fig. 4c shows regions of locking as a function of $d$ and $D$. In regions of locking, a wave is sent out from the central fast pacemaker. The speed of this wave increases with smaller $d$ and larger diffusion. Fig. 4e shows snapshots of the system with three different values of $d$, for a fixed diffusion. The two extremes are clear: small $d$ corresponds to synchronization and waves. This makes sense intuitively, since for $d \rightarrow 0$ the gaps vanish and the pacemakers form an oscillatory medium themselves. For large $d$, all pacemakers oscillate independently, the middle pacemaker a little bit faster than the others. For intermediate values of $d$, the pacemakers are not locked but still they seem to influence each other, leading to irregular patterns such as near-antiphase oscillations as shown in the middle snapshot.

Our results for $\mu=0$ resemble those for systems of discrete oscillators with nearest neighbor coupling. Those systems typically exhibit a transition to synchronization for a critical coupling strength. ${ }^{27,28}$ In our system this coupling strength is determined by the distance and diffusion. Additionally, the gap between pacemakers pro- 
$\mathbf{a}$

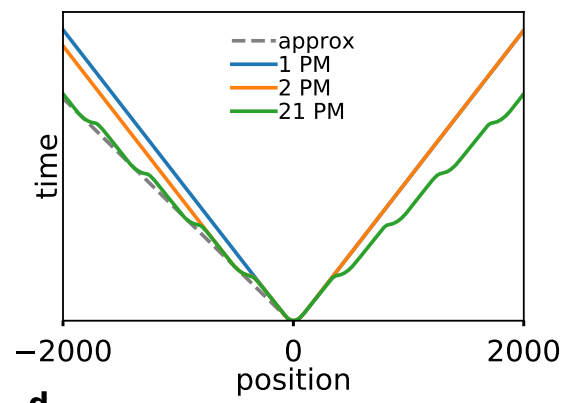

b

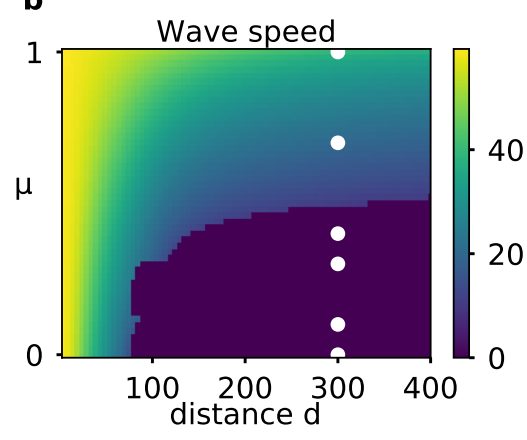

c

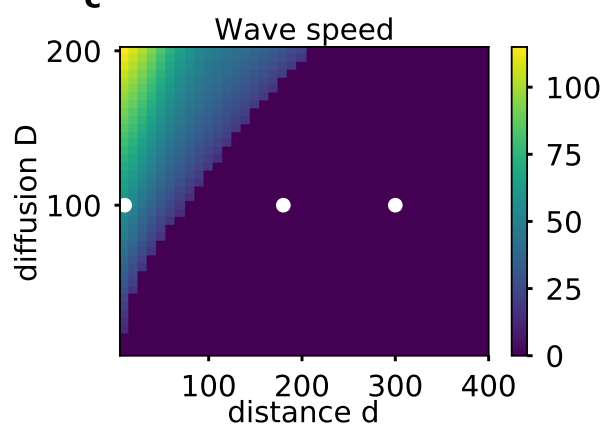

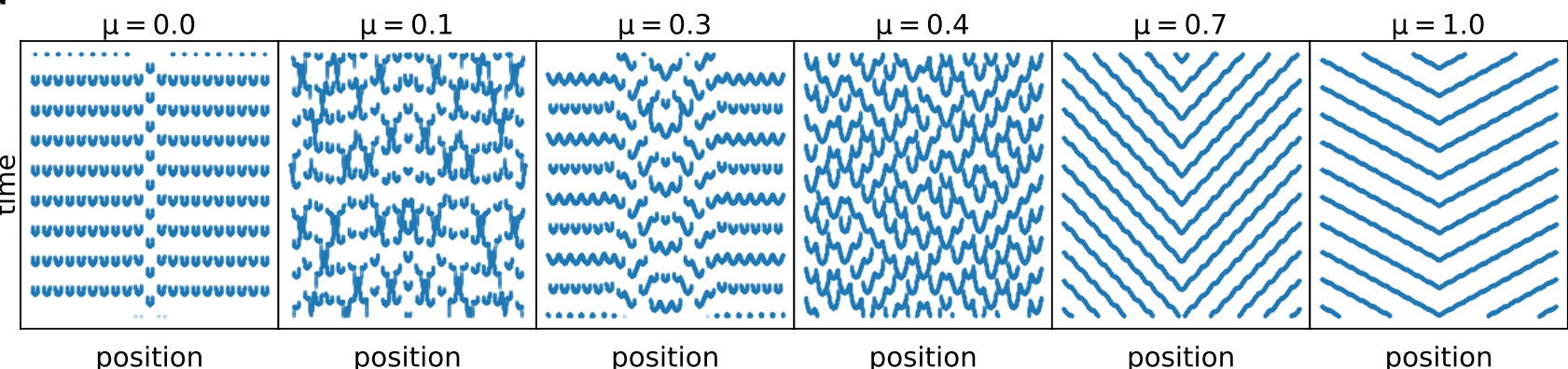

e

$d=10$ $d=180$

$d=300$

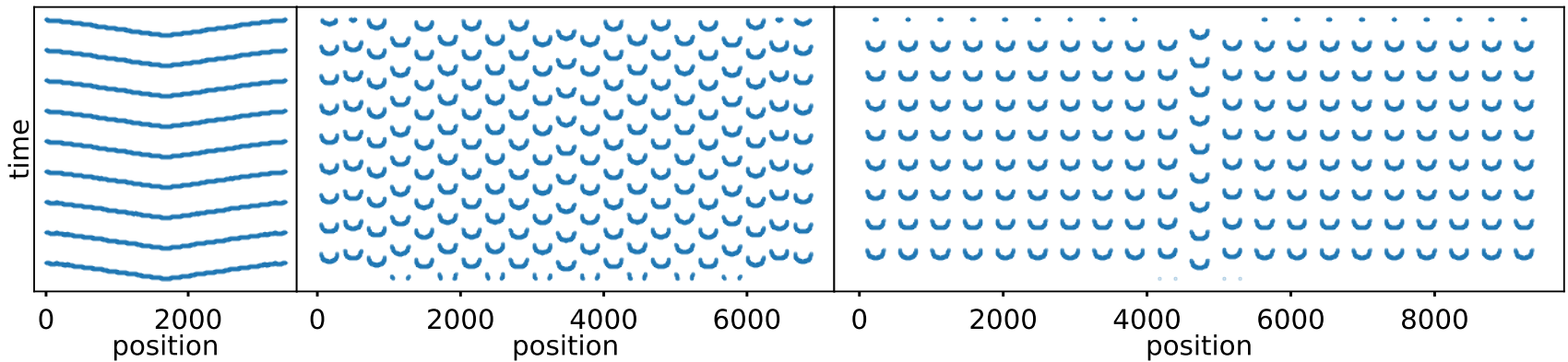

FIG. 4. Analysis for multiple (21) pacemakers. (a) Curves of constant phase for one (blue), two (orange) and 21 (green) pacemakers. Dashed line gives the estimation for 21 pacemakers, assuming $n$ 'repeaters' will speed up the signal $n$ times more than one repeater. (b) Wave speed as function of $d$ and $\mu$, with parameter values $p=1.5, w=150$ and $D=100$. In the blue region, the pacemakers are not synchronized and therefore there is no wave. (c) Wave speed as function of $d$ and $D$, with parameter values of $p=1.5, w=150$ and $\mu=0$. Again, in the blue region there is no synchronization. (d) Points of constant phase $(u=0)$ in the $(x, t)$ plane. Pacemakers have widths $w=150$, other parameters are $p=1.5, d=300$ and $D=100$. Different plots for different values of $\mu$, coinciding with the white dots in (b). (e) Similar as (d) but for fixed $\mu=0$ and changing distance (i.e. different domain sizes $L$ ). Figures corresponding to the white dots in (c).

vides a time delay in the coupling, since $u$ and $v$ have to diffuse through the gap in order to influence the neighboring pacemaker. Systems of discrete coupled oscillators with and without delay show a wealth of different types of behavior. ${ }^{27,29,30}$ Some of this behavior, such as locking and antiphase oscillations, is also seen in models where the coupling is indirect, such as through a bath. ${ }^{31}$ An avenue for future research is thus to map our spatially extended system to a system of discrete oscillators where coupling strength and time delay depend on $d$ and $D$ and compare their behavior.

The transition to synchrony for decreasing distances has already been reported experimentally in systems that strongly resemble our dynamical system. For example, in microfluidic devices that produce droplets of $\mathrm{BZ}$ reaction mixture separated by oil, the inter-droplet distance can be modified. ${ }^{18} \mathrm{~A}$ transition to synchrony happens when this distance is small enough. Similar results are seen in the artificial cell system of Tayar et al. ${ }^{20}$

\section{DISCUSSION}

Oscillatory media have been under scrutiny for a few decades now, yet a lot of questions remain. In our study we have explored the effect of multiple competing pacemakers in the same medium. It is known that the strongest pacemaker entrains the whole medium, where the strength of a pacemaker depends on the size and 
frequency difference of the pacemaker, and on the nature and dispersion of the medium. ${ }^{8,9,22}$ In this paper, we have explicitly shown which properties lead a pacemaker to be the dominant one in a medium composed of FitzHugh-Nagumo oscillators. We have shown how the size and the frequency difference lead a faster oscillatory region to entrain the rest of the medium. Even after this fast pacemaker has taken over the whole medium, the presence of weaker pacemakers still influences the waves. We have quantified how the wave speed depends on the pacemaker's characteristics, and how it is influenced by additional pacemakers. A second strand of our study was aimed at pacemakers separated by regions in which no local dynamics occur. The main result here is that pacemakers may synchronize, depending on diffusion and distance. In transition regions, different types of dynamics are seen. Both the transition and the more exotic types of behavior are reminiscent of results obtained for discrete coupled oscillators.

Our results can be relevant for the study of bio(chemical) systems in which oscillation and synchronization play an important role. The main messages can be summarized as follows. Stronger pacemakers, defined by their size and intrinsic frequency, coordinate the system via waves. The speed of those waves, however, becomes smaller as the pacemakers become stronger. Having smaller additional pacemakers in the system helps to relay the signal and speed up its transmission. Waves are fastest in fully oscillatory media, but can also exist if there are small gaps without local dynamics.

A number of extensions of the current study come to mind. First of all, we have always looked at the long-time behavior, when the dynamics have converged. One could also consider initial transient behavior, which is especially relevant for biological systems. For example, how fast does the dominant pacemaker take over the whole medium? For $\mu=0$, the gaps between the pacemaker regions do not have local dynamics but only allow passive diffusion. An extension of the situation we studied here could consider gaps with dynamics which are not oscillatory, but for example excitable or bistable. Excitable media can transfer information much more quickly than diffusion, by propagating fronts. ${ }^{32,33}$ Additionally, the amplitude of a propagating signal does not decrease, whereas in a diffusion-only system the signal amplitude decreases very quickly with distance. For such systems, some kind of delayed coupling is expected to exist even for large gaps between the oscillatory regions. Experimentally some of the results we obtained have been seen in beads and droplets containing the BZ reactants. The use of microfluidic devices is promising to explore more complicated geometries. ${ }^{34}$ It would be interesting to see a (biological) system in which we can test some of the predictions from the models.

Our system with pacemaker regions separated by gaps with no local dynamics is one of the simplest examples of a model which takes into account different types of dynamics in different regions of space. Especially in bi- ology, systems such as cells are anything but homogeneous. Cells contain for example a nucleus, organelles, membranes, cytoplasm, each of which may have different dynamics. Whereas spatial systems with an inhomogeneity, such as the oscillatory media we studied, are a useful first step, mathematical modeling of those systems is also moving towards more hybrid-like models. In such models, different types of structure can have different types of mathematical description. These are then coupled by diffusion and appropriate boundary conditions. For example, Gomez-Marin et al. ${ }^{35}$ study a simple system of two membranes coupled by a medium. In this medium, chemicals can only diffuse and degrade. On the membranes, the system is modeled by an ODE with FitzHughNagumo dynamics. The membrane dynamics are not oscillatory in isolation, but both in-phase and antiphase oscillations on both ends are induced by the spatial coupling. Similar systems have been mathematically investigated by, among others, Gou, Ward and colleagues. ${ }^{36}$ In those models, compartments modeled by ODEs are connected by a bulk medium modeled by a PDE. A major difference with our current study is the fact that here pacemaker regions are oscillatory, whereas in the cited models the oscillations are induced by the spatial coupling. As a final example, Naqib et. al studied a onedimensional reaction-diffusion system in which chemical species can only be synthesized at two places, and can diffuse from these places. ${ }^{37}$ Interestingly, modifying the spatial separation can induce a range of different dynamics in this system.

Finally we would like to remark on a question which until now we have not touched upon: where do the inhomogeneities, which locally increase oscillation frequency, come from? For target patterns observed in the BZ reaction, it is usually assumed that these come from pieces of dust or scratches in the dish. For biological systems however, they either come from spatial structure as discussed in the previous paragraph, or through self-organization such as in colonies of Dictyostelium discoideum. Mathematical models of biological systems that combine oscillatory dynamics with the formation of pacemakers are rare $^{38,39}$ but can possibly shed light on many observed phenomena.

\section{ACKNOWLEDGMENTS}

This work was triggered by scientific discussions at the seventeenth workshop on instabilities and nonequilibrium structures in Valparaiso, Chile (December 2-6 2019). This workshop was held on the occasion of Enrique Tirapegui's 80th birthday. Unfortunately, Enrique passed away shortly afterwards, in March 2020. We would like to devote this article to Enrique and thank him for his invaluable contributions to science. From Leuven (Belgium), where Enrique spent several years of his career about 40 years ago, our heartfelt condolences go out to his family and friends. 
This work was supported by the Research Foundation - Flanders for individual support (J.R.) and project support (grant GOA5317N), and the KU Leuven Research Fund (C14/18/084).

The computational resources and services used in this work were provided by the VSC (Flemish Supercomputer Center), funded by the Research Foundation - Flanders (FWO) and the Flemish Government - department EWI.

\section{DATA AVAILABILITY}

The data and numerical codes used to generate the data in this study are available from the corresponding author upon reasonable request.

\section{REFERENCES}

${ }^{1}$ A. M. Zhabotinsky and A. N. Zaikin, "Autowave processes in a distributed chemical system," Journal of Theoretical Biology 40, 45-61 (1973)

${ }^{2}$ S. Jakubith, H. H. Rotermund, W. Engel, A. von Oertzen, and G. Ertl, "Spatiotemporal concentration patterns in a surface reaction: Propagating and standing waves, rotating spirals, and turbulence," Physical Review Letters 65, 3013-3016 (1990).

${ }^{3}$ C. Punckt, M. Stich, C. Beta, and H. H. Rotermund, "Suppression of spatiotemporal chaos in the oscillatory $\mathrm{CO}$ oxidation on Pt(110) by focused laser light," Physical Review E 77, 046222 (2008).

${ }^{4}$ K. J. Lee, E. C. Cox, and R. E. Goldstein, "Competing Patterns of Signaling Activity in Dictyostelium Discoideum," Physical Review Letters 76, 1174-1177 (1996).

${ }^{5}$ J. B. Chang and J. E. Ferrell, "Mitotic trigger waves and the spatial coordination of the Xenopus cell cycle," Nature 500, 603607 (2013).

${ }^{6}$ G. Bub, A. Shrier, and L. Glass, "Global Organization of Dynamics in Oscillatory Heterogeneous Excitable Media," Physical Review Letters 94, 028105 (2005).

${ }^{7}$ A. Pikovsky, J. Kurths, M. Rosenblum, and J. Kurths, Synchronization: A Universal Concept in Nonlinear Sciences (Cambridge University Press, 2003)

${ }^{8}$ P. S. Hagan, "Target patterns in reaction-diffusion systems," Advances in Applied Mathematics 2, 400-416 (1981).

${ }^{9}$ Y. Kuramoto, Chemical oscillations, waves and turbulence (Springer-Verlag, Berlin, 1984).

${ }^{10}$ A. S. Mikhailov and A. Engel, "Multiple target pattern creation and synchronization phenomena," Physics Letters A 117, 257260 (1986).

${ }^{11} \mathrm{R}$. Tönjes and B. Blasius, "Perturbation analysis of the $\mathrm{Ku}$ ramoto phase-diffusion equation subject to quenched frequency disorder," Physical Review E - Statistical, Nonlinear, and Soft Matter Physics 79, 1-9 (2009).

${ }^{12} \mathrm{~B}$. Novák and J. J. Tyson, "Design principles of biochemical oscillators," Nature Reviews Molecular Cell Biology 9, 981-991 (2008).

${ }^{13}$ A. E. Bugrim, M. Dolnik, A. M. Zhabotinsky, and I. R. Epstein, "Heterogeneous sources of target patterns in reactiondiffusion systems," Journal of Physical Chemistry 100, 1901719022 (1996)

${ }^{14}$ V. E. Deneke, A. Puliafito, D. Krueger, A. V. Narla, A. De Simone, L. Primo, M. Vergassola, S. De Renzis, and S. Di Talia, "Self-Organized Nuclear Positioning Synchronizes the Cell Cycle in Drosophila Embryos," Cell 177, 925-941.e17 (2019).
${ }^{15}$ O. U. Kheowan, E. Mihaliuk, B. Blasius, I. Sendiña-Nadal, and K. Showalter, "Wave mediated synchronization of nonuniform oscillatory media," Physical Review Letters 98, 2-5 (2007).

${ }^{16}$ A. F. Taylor, M. R. Tinsley, F. Wang, Z. Huang, and K. Showalter, "Dynamical Quorum Sensing and Synchronization in Large Populations of Chemical Oscillators," Science 323, 614-617 (2009).

${ }^{17} \mathrm{R}$. Toth and A. F. Taylor, "Loss of coherence in a population of diffusively coupled oscillators," The Journal of Chemical Physics 125, 224708 (2006)

${ }^{18}$ H. Fukuda, N. Tamari, H. Morimura, and S. Kai, "Entrainment in a chemical oscillator chain with a pacemaker," Journal of Physical Chemistry A 109, 11250-11254 (2005).

${ }^{19}$ N. Li, J. Delgado, H. O. González-Ochoa, I. R. Epstein, and S. Fraden, "Combined excitatory and inhibitory coupling in a 1 d array of Belousov-Zhabotinsky droplets," Physical Chemistry Chemical Physics 16, 10965-10978 (2014).

${ }^{20}$ A. M. Tayar, E. Karzbrun, V. Noireaux, and R. H. Bar-Ziv, "Synchrony and pattern formation of coupled genetic oscillators on a chip of artificial cells," Proceedings of the National Academy of Sciences 114, 11609-11614 (2017).

${ }^{21}$ H. Kitahata, J. Taguchi, M. Nagayama, T. Sakurai, Y. Ikura, A. Osa, Y. Sumino, M. Tanaka, E. Yokoyama, and H. Miike, "Oscillation and Synchronization in the Combustion of Candles," The Journal of Physical Chemistry A 113, 8164-8168 (2009).

${ }^{22}$ M. Stich and A. S. Mikhailov, "Complex Pacemakers and Wave Sinks in Heterogeneous Oscillatory Chemical Systems," Zeitschrift fr Physikalische Chemie 216, 521 (2002).

${ }^{23}$ X. Shao, Y. Wu, J. Zhang, H. Wang, and Q. Ouyang, "Inward Propagating Chemical Waves in a Single-Phase ReactionDiffusion System," Physical Review Letters 100, 198304 (2008), publisher: American Physical Society.

${ }^{24}$ R. FitzHugh, "Impulses and Physiological States in Theoretical Models of Nerve Membrane," Biophysical Journal 1, 445-466 (1961).

${ }^{25}$ J. Nagumo, S. Arimoto, and S. Yoshizawa, "An Active Pulse Transmission Line Simulating Nerve Axon," Proceedings of the IRE 50, 2061-2070 (1962).

${ }^{26}$ M. Stich and A. S. Mikhailov, "Target patterns in twodimensional heterogeneous oscillatory reactiondiffusion systems," Physica D: Nonlinear Phenomena 215, 38-45 (2006)

${ }^{27}$ Z. Zheng, G. Hu, and B. Hu, "Phase Slips and Phase Synchronization of Coupled Oscillators," Physical Review Letters 81, 5318-5321 (1998)

${ }^{28}$ F. Radicchi and H. Meyer-Ortmanns, "Entrainment of coupled oscillators on regular networks by pacemakers," Physical Review E 73, 036218 (2006), publisher: American Physical Society.

${ }^{29}$ S. A. Campbell and Z. Wang, "Phase models and clustering in networks of oscillators with delayed coupling," Physica D: Nonlinear Phenomena 363, 44-55 (2018).

${ }^{30}$ R. Dodla, A. Sen, and G. L. Johnston, "Phase-locked patterns and amplitude death in a ring of delay-coupled limit cycle oscillators," Physical Review E 69, 056217 (2004).

${ }^{31}$ E. Camacho, R. Rand, and H. Howland, "Dynamics of two van der Pol oscillators coupled via a bath," International Journal of Solids and Structures 41, 2133-2143 (2004).

${ }^{32}$ J. J. Tyson and J. P. Keener, "Singular perturbation theory of traveling waves in excitable media (a review)," Physica D: Nonlinear Phenomena 32, 327-361 (1988).

${ }^{33}$ L. Gelens, G. A. Anderson, and J. E. Ferrell, "Spatial trigger waves: positive feedback gets you a long way," Molecular Biology of the Cell 25, 3486-3493 (2014).

${ }^{34}$ K. Torbensen, F. Rossi, S. Ristori, and A. Abou-Hassan, "Chemical communication and dynamics of droplet emulsions in networks of BelousovZhabotinsky micro-oscillators produced by microfluidics," Lab on a Chip 17, 1179-1189 (2017).

${ }^{3}$ A. Gomez-Marin, J. Garcia-Ojalvo, and J. M. Sancho, "SelfSustained Spatiotemporal Oscillations Induced by MembraneBulk Coupling," Physical Review Letters 98, 168303 (2007). 
${ }^{36}$ J. Gou, W.-Y. Chiang, P.-Y. Lai, M. J. Ward, and Y.-X. Li, "A theory of synchrony by coupling through a diffusive chemical signal," Physica D: Nonlinear Phenomena 339, 1-17 (2017).

${ }^{37}$ F. Naqib, T. Quail, L. Musa, H. Vulpe, J. Nadeau, J. Lei, and

L. Glass, "Tunable oscillations and chaotic dynamics in systems with localized synthesis," Physical Review E - Statistical, Non- linear, and Soft Matter Physics 85, 1-8 (2012).

${ }^{38}$ A. S. Mikhailov, "Stable autonomous pacemakers in the enlarged Ginzburg-Landau model," Physica D 5, 99-112 (1992).

${ }^{39}$ H. Sakaguchi and S. Maeyama, "Dynamical quorum sensing and clustering dynamics in a population of spatially distributed active rotators," Physical Review E 87, 024901 (2013). 

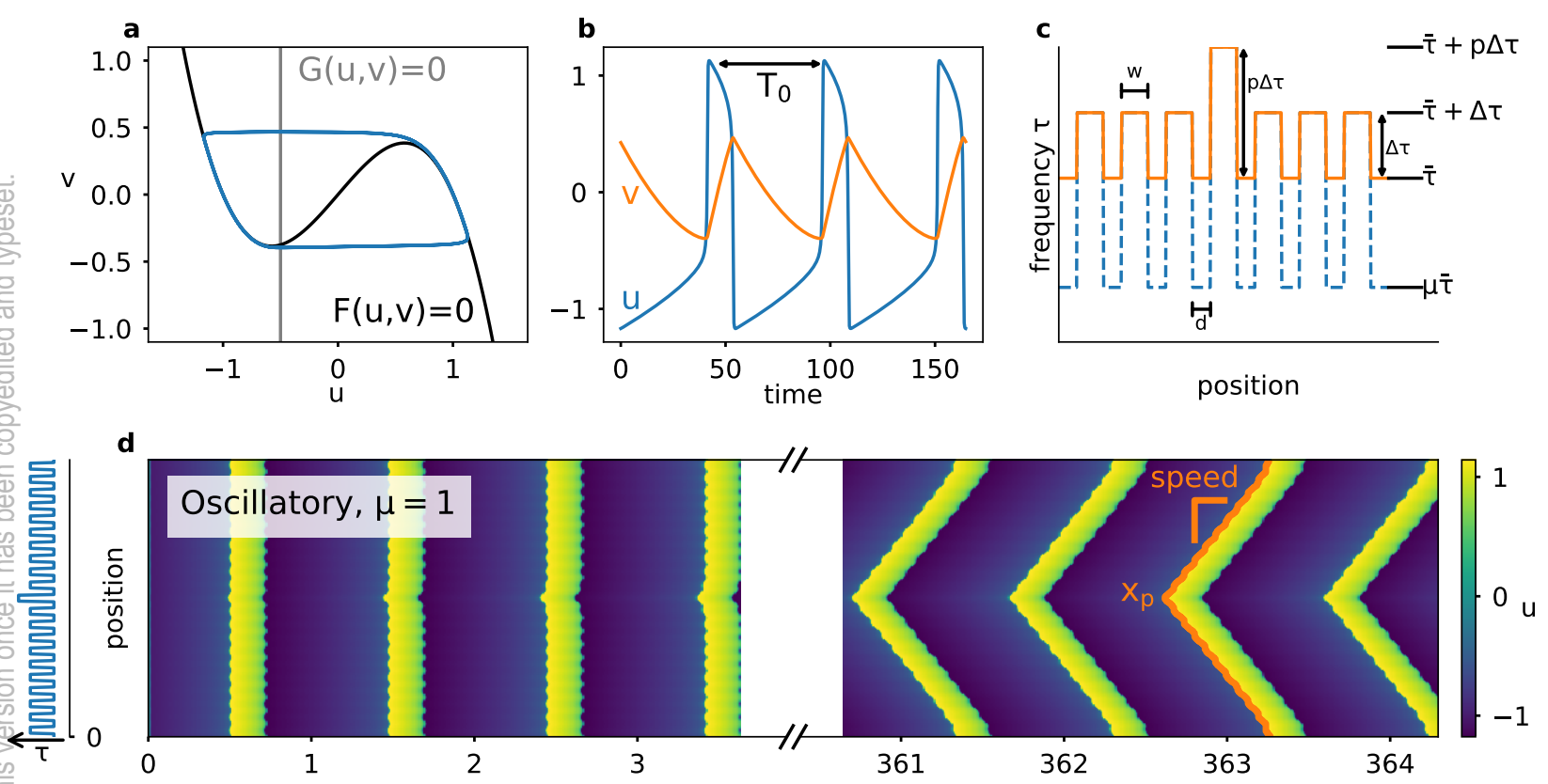

:
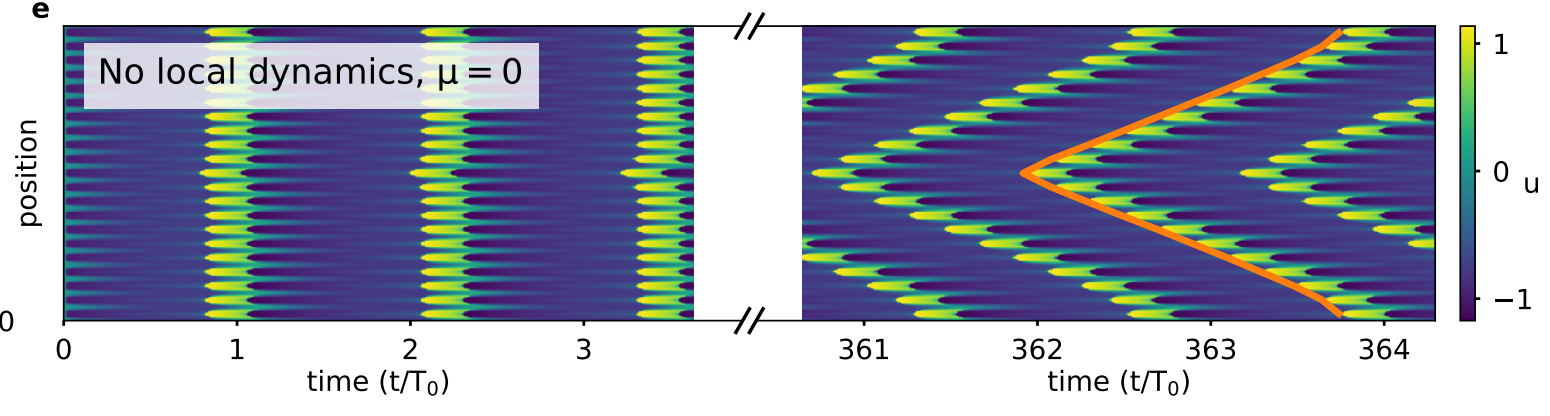

등

일

安

드는

辛紫

文

오 뜬

문

衣

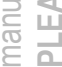




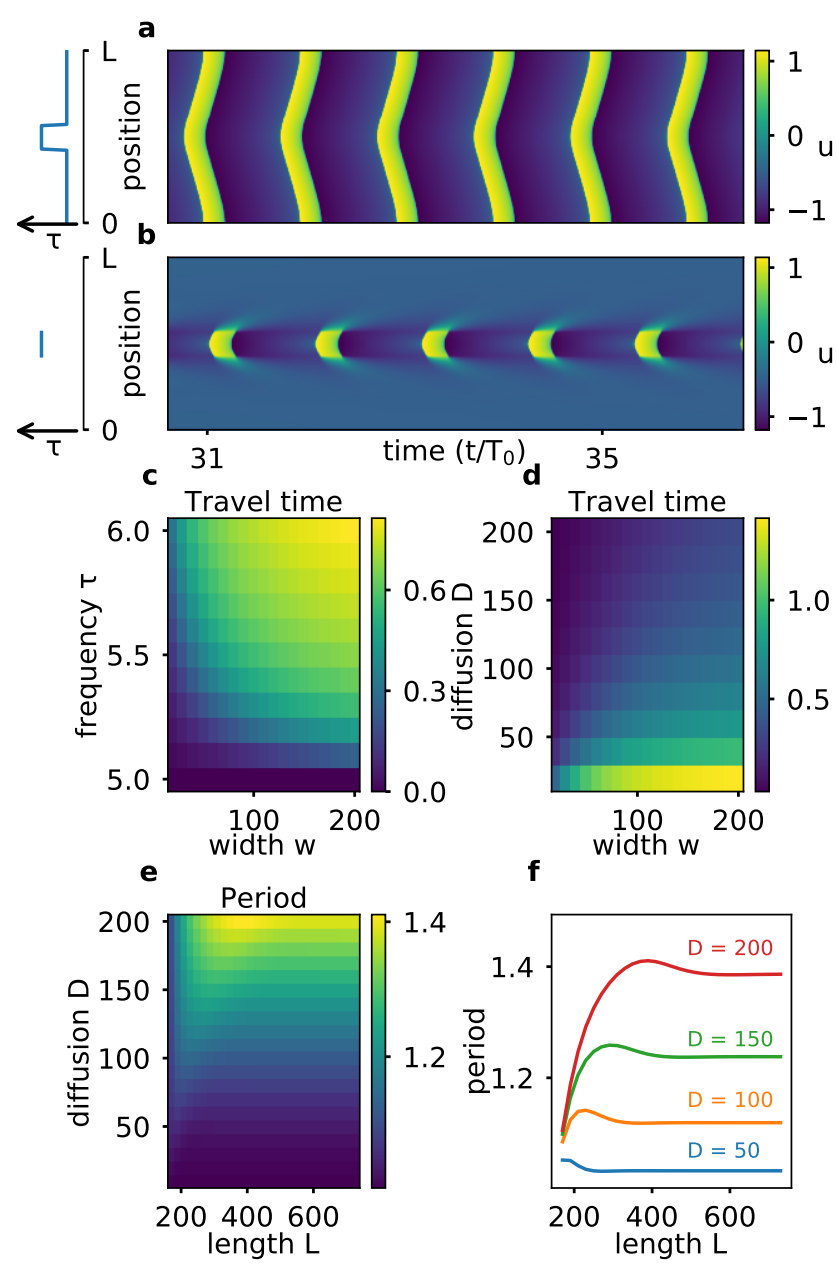


